\title{
Successful Pregnancy Following Dual Triggering Concept (rhCG + GnRH Agonist) in a Patient Showing Repetitive Inmature Oocytes and Empty Follicle Syndrome: Case Report
}

\author{
Juan Carlos Castillo ${ }^{\mathrm{a}, \mathrm{c}}$, Joaquin Moreno ${ }^{\mathrm{a}}$, Miguel Dolz ${ }^{\mathrm{a}}$, Fernando Bonilla-Musoles ${ }^{\mathrm{b}}$
}

\begin{abstract}
After ovarian stimulation some oocytes collected for IVF cycles are arrest at the GV o MI stage at the time of retrieval despite controlled ovarian stimulation (COS) and a correct administration of hCG. However, it is unusual to have a complete failure of oocyte maturation after COS, especially in presence of normally growing follicles. Empty follicle syndrome (EFS) is a condition of uncertain etiology in which no oocytes are retrieved from apparently normally growing ovarian follicles with normal estradiol levels after ovarian stimulation for assisted reproductive techniques. In genuine EFS, dysfunction of the folliculogenesis seems to be the most plausible etiology. We report a case of primary infertility in which four consecutive IVF attempts yielded immature oocytes or EFS. Finally, at a fifth attempt the use of hCGr + GnRHa (dual triggering) for final follicular maturation resulted in a successful live birth.
\end{abstract}

Keywords: IVF; Immature oocytes; Empty follicle syndrome; GnRH agonist; hCG

\section{Introduction}

Controlled ovarian stimulation (COS) is aimed to induce multiple follicular growth. Oocyte maturation for IVF cycles is commonly induced by hCG (as surrogate of natural LH surge). Following LH surge, the resumption of meiosis occurs about 18 hours later and achievement of second metaphase occurs within 28 to 38 hours [1]. After ovarian stimulation (and probably due to multifollicular development)

\footnotetext{
Manuscript accepted for publication January 3, 2013

${ }^{\mathrm{a}}$ Grupo Fiv Valencia. Calle Santa Rosa 12, 46021. Valencia. Spain

${ }^{b}$ Hospital Clinico Universitario de Valencia. Avenida Blasco Ibanez, 17, 46010 Valencia. Spain

${ }^{\mathrm{c}}$ Corresponding author: Juan Carlos Castillo. Grupo Fiv Valencia, Calle

Santa Rosa 12, 46021. Valencia. Spain.

Email: jccastillof@hotmail.com
}

doi: http://dx.doi.org/10.4021/jmc1055w some oocytes collected for IVF cycles are arrest at the GV o MI stage at the time of retrieval despite COS and a correct administration of hCG [2]. However, it is unusual to have a complete failure of oocyte maturation after COS, especially in presence of normally growing follicles.

Empty follicle syndrome (EFS) is a condition of uncertain etiology in which no oocytes are retrieved from apparently normally growing ovarian follicles with normal estradiol levels after ovarian stimulation for assisted reproductive techniques (ART). Several hypotheses as to the background of EFS after hCG triggering of final oocyte maturation in gonadotropin stimulated cycles have been summarized elsewhere recently [3]. In genuine EFS, dysfunction of the folliculogenesis seems to be the most plausible etiology $[4,5]$.

Since the mid-90s -following the availability of GnRH antagonist for clinical practice- the use of a bolus of $\mathrm{GnRH}$ agonist (GnRHa) for final follicular maturation has been explored in many clinical scenarios [6]. Among the advantages suggested for $\mathrm{GnRHa}$ as trigger agent are: the induction of a more physiological way of triggering resembling a natural cycle (release of LH and FSH); higher number of oocytes MII collected [7-10] and OHSS prevention. More recently, Shapiro et al described the so-called "dual trigger" which involves the triggering of final oocyte maturation using a combination of a GnRHa together with a low-dose hCG for IVF cycles $[11,12]$ for OHSS high risk patients under COS.

Levran et al (2002) [13] described 8 cases of oocyte maturation arrest and more recently Beck-Fruchter et al (2012) [14] described EFS treatment including a complete review of the literature. However, our group is not aware of any publication showing both anomalies: oocyte maturation and EFS occurring in the same patient as in the case described herein.

We report a case of primary infertility in which four consecutive IVF attempts yielded immature oocytes or EFS. Finally at a fifth attempt the use of hCGr + GnRHa (dual triggering) for final follicular maturation resulted in a successful live birth.

\section{Case Report}

A 37-year-old woman with a 3-year history of primary infer- 
tility was referred to our IVF unit for unexplained infertility. She underwent a thorough infertility investigation without any abnormal findings identified. She was in good general health and with no family history of infertility. No history of exposure to any medications for other medical conditions during or prior IVF, she also did not have past exposure to environmental or occupational toxicants and/or excessive Xray radiation. She had a normal female kariotype $(46, \mathrm{XX})$ and normal levels of day 3 FSH (6 UI/L), LH (3 UI/L) and estradiol $(28 \mathrm{pg} / \mathrm{mL})$ levels at first IVF attempt. Her husband's sperm showed: volume $2.5 \mathrm{~mL}$, count: 16 mill/mL, motility $40 \%, 20 \%$ of normal morphology.

The patient underwent $\mathrm{COS}$ according to our routine IVF program. In brief, long GnRH agonist protocol (leu-

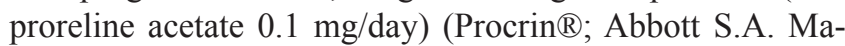
drid, Spain) followed by rFSH 200 - 250 IU/day (Gonal-F®; Merck-Serono Laboratories; A. Madrid, Spain). Ovarian response was monitored by endovaginal scanning and rhCG (Ovitrelle ${ }^{\circledR}$ Serono Laboratories; A. Madrid, Spain) was given when there were at least 2 follicles $>16 \mathrm{~mm}$ of maximum diameter. The serum E2 was measured on the day of hCG administration. Oocyte retrieval was scheduled 36 hours after triggering at $1 \mathrm{st}$ attempt, while on 2 nd to 4 th attempts the hCG-oocyte retrieval interval was prolonged to 40 hours. Finally, at 5 th attempt, an antagonist flexible multiple dose protocol was used, starting Cetrotide ${ }^{\circledR}$; (Serono International S.A. Geneva, Switzerland) $0.25 \mathrm{mg}$ /day when the leading follicle was $14 \mathrm{~mm}$ in diameter; final follicular maturation was done using Ovitrelle ${ }^{\circledR}$ (Serono Laboratories; A. Madrid, Spain) plus triptoreline $0.2 \mathrm{mg}$ (Decapeptyl $\AA^{\circledR}$, IPSEN PHARMA S.A. Barcelona, Espana) via abdominal subcutaneous route at the same moment but using different zones for administration and the oocyte pick up scheduled for 36 hours later. While in the initial four attempts there were no oocytes or only MI oocytes collected, at 5 th attempt 8 oocytes were retrieved. Assuming an unexplained "oocyte factor" four oocytes were striped of cumulus cells for the ICSI procedure whereas the others were inseminated using conventional IVF. Five good quality embryos [15] were obtained on day +3 ( 3 from ICSI and 2 from IVF); 3 embryos ( 1 from ICSI and 2 from IVF) were transferred under ultrasound guidance. Two surplus embryos were frozen. Luteal support was as usual using micronized progesterone $600 \mathrm{mg} /$ day (Progeffik ${ }^{\circledR}$ EFFIK Laboratories, S.A. Madrid, Spain). The patient conceived and delivered healthy baby $(3,700 \mathrm{~g})$ at term by vaginal route.

Stimulation and laboratory finding are summarized in Tables 1 and 2.

\section{Discussion}

Oocyte maturation arrest is a complex problem not clearly understood; only few cases showing a complete oocyte

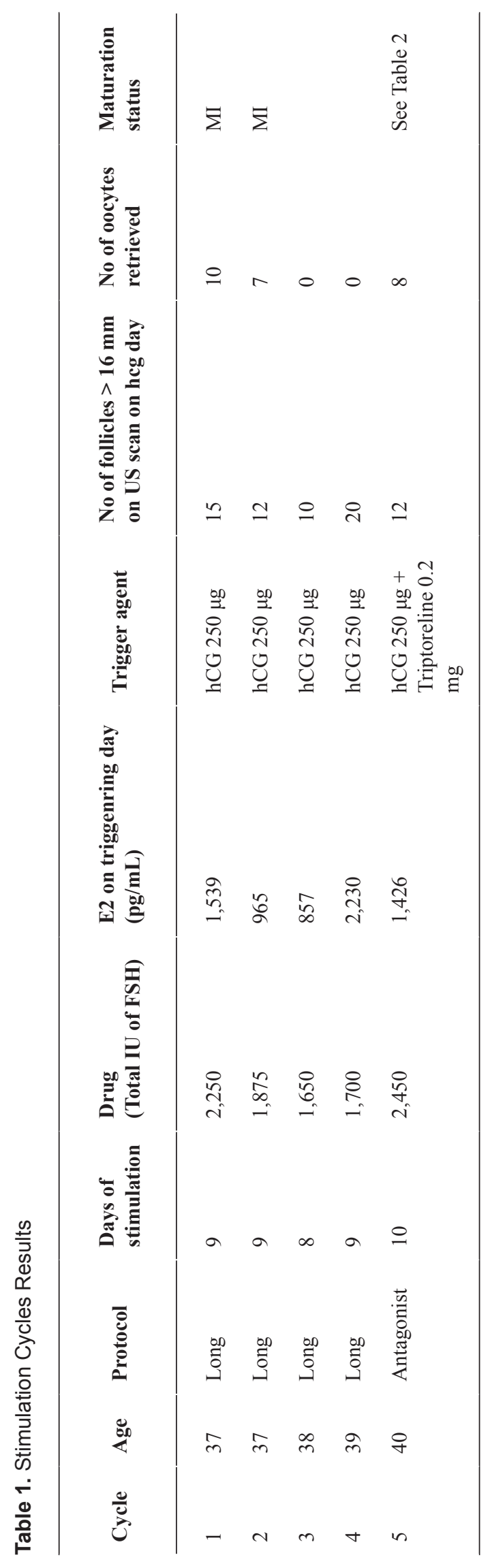


Table 2. Laboratory Results at 5th Cycle

\begin{tabular}{|c|c|c|c|c|c|c|}
\hline \multirow{2}{*}{$\begin{array}{l}\text { Oocytes } \\
\text { retrieved }\end{array}$} & \multicolumn{2}{|c|}{ Maturation status } & \multicolumn{2}{|c|}{ Fertilization } & \multicolumn{2}{|c|}{ Day +3} \\
\hline & IVF & ICSI & Polar body & Pronucleous & Cells & Grading \\
\hline 1 & & MII & 2 & 2 & 8 & $\mathrm{~A}^{2}$ \\
\hline 2 & & MII & 2 & 2 & 8 & $\mathrm{~A}^{2}$ \\
\hline 3 & & MII & 2 & 2 & 8 & $\mathrm{~A}^{1}$ \\
\hline 4 & & MII & 1 & 0 & & \\
\hline 5 & 2 & & 2 & 2 & 8 & $\mathrm{~A}^{1}$ \\
\hline 6 & 2 & & 2 & 2 & 8 & $\mathrm{~A}^{1}$ \\
\hline 7 & 3 & & 1 & 0 & & \\
\hline 8 & 3 & & 1 & 0 & & \\
\hline
\end{tabular}

${ }^{1}$ Embryos transferred; 'Frozen embryos; Embryo grading criteria according to ASEBIR.

maturation failure has been reported previously, still the exact incidence remains unknown $[13,16]$. Failure to resume meiosis in vivo may arise at one of the following three levels: (1) absent or incomplete LH surge; (2) derangements in the signaling mechanisms from the surrounding cumulus cells; and (3) intrinsic oocyte factor [13]. Specifically, metaphase I arrest is suggested to occur due to (a) when collected, oocytes still did not attain the full competence to mature; (b) this arrest may result from the absence of meiotic recombination, which, under normal conditions, occurs in pachytene stage; and (c) may be in an inability of oocytes to produce the key cell cycle regulating factors [17]. EFS is also a condition of uncertain etiology. The incidence of EFS after hCG triggering in IVF patients has been estimated to be about $2-7 \%$ [18]. The so called "genuine EFS" is presumably related to intrinsic ovarian factors and an altered folliculogenesis [3-5].

In the natural cycle the responsiveness of the preovulatory follicle to gonadotropins is maximum, especially (but not only) to luteinizing hormone (LH) that triggers granulosa wall dissociation and cumulus expansion as well as oocyte nuclear maturation [19]. Nonetheless, we must remember that the oocyte itself do not respond to FSH and LH exposure [20-23] since oocytes lack gonadotrophin receptors [20, 24]; hence, the stimulation of meiotic resumption by gonadotrophins occurs via their actions on the surrounding somatic cells rather than on the oocyte itself [25]. Finally, we may assume that any failure (possibly at the biochemical level) in the signaling mechanisms controlling the interaction be- tween cumulus cells and the oocyte may cause oocyte maturation arrest or lack of cumulus expansion/granulose cells dissociation, which -at least in animals models- correlates with the outcome of oocyte maturation [26].

Interestingly, the protocol used in cases previously reporting MI arrest was the long protocol [13]. The same happened in our case, the fourth initial attempts were conducted under the long agonist protocol. At the biochemical level, granulose lutein cells have showed a different pathway modulation of the FHS-induced kinases in agonist versus antagonist cycles [27]; hence it is logical to assume that the type of protocol may influence intrafollicular environment and oocyte maturation pathways and may contribute in some cases to abnormal oocyte development. However, other findings challenge this hypothesis, for example EFS cases are described in both agonist and antagonist protocols and also when GnRHa are used for triggering [3]. So the influence of the type of protocol to EFS or oocyte maturation arrest remains still inconclusive, but not negligible.

In the natural cycle, the resumption of oocyte meiotic maturation and ovulation process is controlled by the midcycle secretion gonadotrophins by the hypophysis; this preovulatory gonadotrophin surge is thought to be the primary stimulus by which oocyte maturation is reinitiated [13]. In vivo, this surge lasts about 48 hours. In ART a bolus of hCG is commonly used as a surrogate of the midcycle surge LH. There are, however, important differences; the half-life of $\mathrm{hCG}$ is much longer (days) than that of the natural cycle (hours); but, and more importantly for this case, the natural 
midcycle also includes a FSH peak which is known, -among other functions-, to promote nuclear maturation of the oocytes. In a GnRH antagonist cycle a bolus of a GnRHa can be administered, as an alternative to hCG for the induction of an endogenous mid-cycle surge (the flare up effect), consisting of both LH and FSH. It has been demonstrated that is as effective as hCG for inducing a correct final follicular maturation [6]; moreover the duration of the GnRHa surge, is closer to that of the natural cycle [28]. However, as seen in the majority of IVF cycles this FSH surge is not always needed, as hCG can efficiently act as a surrogate of LH in promoting final follicular maturation. However, we may assume that in some patients, like our case, this FSH surge may promote an adequate resumption of meiosis or optimize the signaling within cumulus cells preventing the occurrence of EFS and also inducing oocyte maturation until MII stage. Clinical evidence of this statements are provided by medical literature, for example some cases of EFS are solved by changing the trigger agent to GnRH agonist [14, 29], furthermore, the retrieval of more mature oocytes after $\mathrm{GnRHa}$ triggering observed in IVF cycles supports a possible beneficial effect of the mid-cycle FSH surge on oocyte maturity [7-10].

Optimal levels of hCG for a final follicular maturation are still not defined; in the present case hCG levels were not measured, therefore we cannot exclude the possibility of false or borderline EFS. However, in view of previous bad results after hCG trigger in this patient; we may assume that a GnRHa trigger alone at fifth cycle may have resulted in a adequate oocyte maturation, but, in this case a high response was not expected so we proceed with the dual triggering concept with the knowledge that the introduction of hCG in usual doses will not suppose OHSS risk for this patient, on contrary, the addition of hCG would bring LH activity for luteal support to over count the luteolitic effect seen after GnRHa triggering, strategy that is important for a normalization of pregnancy rates after GnRHa trigger as described recently [30].

In the present case, it is likely that the reason for both EFS and meiotic arrest lie within the cumulus-oocyte complex. Among the findings supporting this hypothesis are: first of all, the repetitive nature of cycle results, on second place the diagnosis of primary infertility which seems to be a typical profile in this cases as described by Levran et al (2002) (seven out of eight of his cases presented primary infertility); also, cycle development was normal showing good follicular development as well as the observation of normal steroidogenesis the triggering day all of which represent normal LH effects, also the ovarian pick up were reported as ease procedures. This hypothesis is line with previous findings which supports the concept of dysfunctional folliculogenesis as the background of EFS in IVF patients (Castillo et al, 2012). Hence, it is possible to assume that a more physiologic induction of final follicular maturation in terms of gonadotrophin surge duration and/or the addition of natural FSH/LH activity directly from de hypophysis may optimize the signaling mechanisms from the surrounding cumulus and the oocyte, resulting -at least for this case- in adequate oocyte retrieval and maturation.

\section{Conclusion}

Although we still do not know the exact pathophysiology of these syndromes, and more research on the physiologic pathways controlling oocyte maturation is needed before the exact mechanisms of the defects interfering with meiotic competence can be determined; the favorable result in the present case gives additional idea for treatment and provides a feasible option to patients with EFS or repetitive immature oocyte syndrome, in the form of a simpler antagonist protocol combined with a more physiologic form of final follicular maturation trough a FSH plus LH surge induced by a bolus of GnRH agonist. Final conclusions should wait for the proof of the approach with more cases.

\section{Authors' Contributions}

JCC analyzed and interpreted the patient data regarding previous IVF attempts; he was in charge of the final procedures in the actual stimulation cycle and a major contributor in designing and writing the manuscript. MD analyzed and interpreted the patient data regarding previous IVF attempts, he was in charge of the actual stimulation cycle and a major contributor in designing the manuscript. JM analyzed and interpreted the patient data regarding previous IVF attempts; he was in charge of gamete/embryo management in the actual cycle and a major contributor in designing the manuscript. FBM analyzed and interpreted the patient data regarding previous IVF attempts; he was a major contributor in designing the manuscript. All authors read and approved the final manuscript.

\section{Declaration}

The authors report no financial or commercial conflicts of interest. The authors declare that they have no competing interests'.

\section{References}

1. Seibel MM, Shine W, Smith DM, Taymor ML. Biological rhythm of the luteinizing hormone surge in women. Fertil Steril. 1982;37(5):709-711.

2. Avrech OM, Goldman GA, Rufas O, Stein A, Amit S, Yoles I, Pinkas H, et al. Treatment variables in relation 
to oocyte maturation: lessons from a clinical micromanipulation-assisted in vitro fertilization program. J Assist Reprod Genet. 1997;14(6):337-342.

3. Castillo JC, Garcia-Velasco J, Humaidan P. Empty follicle syndrome after GnRHa triggering versus hCG triggering in COS. J Assist Reprod Genet. 2012;29(3):249253.

4. Khalaf Y, Braude P. 'Curing' empty follicle syndrome. Hum Reprod. 1997;12(7):1601.

5. Yariz KO, Walsh T, Uzak A, Spiliopoulos M, Duman D, Onalan G, King MC, et al. Inherited mutation of the luteinizing hormone/choriogonadotropin receptor (LHCGR) in empty follicle syndrome. Fertil Steril. 2011;96(2):e125-130.

6. Humaidan P, Kol S, Papanikolaou EG. GnRH agonist for triggering of final oocyte maturation: time for a change of practice? Hum Reprod Update. 2011;17(4):510-524.

7. Imoedemhe DA, Chan RC, Sigue AB, Pacpaco EL, Olazo AB. A new approach to the management of patients at risk of ovarian hyperstimulation in an in-vitro fertilization programme. Hum Reprod. 1991;6(8):10881091.

8. Humaidan P, Bredkjaer HE, Bungum L, Bungum M, Grondahl ML, Westergaard L, Andersen CY. GnRH agonist (buserelin) or hCG for ovulation induction in GnRH antagonist IVF/ICSI cycles: a prospective randomized study. Hum Reprod. 2005;20(5):1213-1220.

9. Humaidan P. Luteal phase rescue in high-risk OHSS patients by GnRHa triggering in combination with low-dose HCG: a pilot study. Reprod Biomed Online. 2009;18(5):630-634.

10. Oktay K, Turkcuoglu I, Rodriguez-Wallberg KA. GnRH agonist trigger for women with breast cancer undergoing fertility preservation by aromatase inhibitor/FSH stimulation. Reprod Biomed Online. 2010;20(6):783-788.

11. Shapiro BS, Daneshmand ST, Garner FC, Aguirre M, Thomas S. Gonadotropin-releasing hormone agonist combined with a reduced dose of human chorionic gonadotropin for final oocyte maturation in fresh autologous cycles of in vitro fertilization. Fertil Steril. 2008;90(1):231-233.

12. Shapiro BS, Daneshmand ST, Garner FC, Aguirre M, Hudson C. Comparison of "triggers" using leuprolide acetate alone or in combination with low-dose human chorionic gonadotropin. Fertil Steril. 2011;95(8):27152717.

13. Levran D, Farhi J, Nahum H, Glezerman M, Weissman A. Maturation arrest of human oocytes as a cause of infertility: case report. Hum Reprod. 2002;17(6):16041609.

14. Beck-Fruchter R, Weiss A, Lavee M, Geslevich Y, Shalev E. Empty follicle syndrome: successful treatment in a recurrent case and review of the literature. Hum Reprod. 2012;27(5):1357-1367.
15. The Istanbul consensus workshop on embryo assessment: proceedings of an expert meeting. Hum Reprod. 2011;26(6):1270-1283.

16. Harrison KL, Sherrin DA, Keeping JD. Repeated oocyte maturation block. J Assist Reprod Genet. 2000;17(4):231-233.

17. Mrazek M, Fulka Jr J, Jr. Failure of oocyte maturation: possible mechanisms for oocyte maturation arrest. Hum Reprod. 2003;18(11):2249-2252.

18. Ben-Shlomo I, Schiff E, Levran D, Ben-Rafael Z, Mashiach S, Dor J. Failure of oocyte retrieval during in vitro fertilization: a sporadic event rather than a syndrome. Fertil Steril. 1991;55(2):324-327.

19. Gougeon A. Human ovarian follicular development: from activation of resting follicles to preovulatory maturation. Ann Endocrinol (Paris). 2010;71(3):132-143.

20. Dekel N, Beers WH. Rat oocyte maturation in vitro: relief of cyclic AMP inhibition by gonadotropins. Proc Natl Acad Sci U S A. 1978;75(9):4369-4373.

21. Eppig JJ. The relationship between cumulus cell-oocyte coupling, oocyte meiotic maturation, and cumulus expansion. Dev Biol. 1982;89(1):268-272.

22. Downs SM, Daniel SA, Eppig JJ. Induction of maturation in cumulus cell-enclosed mouse oocytes by follicle-stimulating hormone and epidermal growth factor: evidence for a positive stimulus of somatic cell origin. J Exp Zool. 1988;245(1):86-96.

23. Byskov AG, Yding Andersen C, Hossaini A, Guoliang $\mathrm{X}$. Cumulus cells of oocyte-cumulus complexes secrete a meiosis-activating substance when stimulated with FSH. Mol Reprod Dev. 1997;46(3):296-305.

24. Amsterdam A, Koch Y, Lieberman ME, Lindner HR. Distribution of binding sites for human chorionic gonadotropin in the preovulatory follicle of the rat. J Cell Biol. 1975;67(3):894-900.

25. Zhang M, Ouyang H, Xia G. The signal pathway of gonadotrophins-induced mammalian oocyte meiotic resumption. Mol Hum Reprod. 2009;15(7):399-409.

26. Yokoo M, Sato E. Cumulus-oocyte complex interactions during oocyte maturation. Int Rev Cytol. 2004;235(251291.

27. Khalaf M, Mittre H, Levallet J, Hanoux V, Denoual C, Herlicoviez M, Bonnamy PJ, et al. GnRH agonist and $\mathrm{GnRH}$ antagonist protocols in ovarian stimulation: differential regulation pathway of aromatase expression in human granulosa cells. Reprod Biomed Online. 2010;21(1):56-65.

28. Itskovitz J, Boldes R, Levron J, Erlik Y, Kahana L, Brandes JM. Induction of preovulatory luteinizing hormone surge and prevention of ovarian hyperstimulation syndrome by gonadotropin-releasing hormone agonist. Fertil Steril. 1991;56(2):213-220.

29. Lok F, Pritchard J, Lashen H. Successful treatment of empty follicle syndrome by triggering endogenous $\mathrm{LH}$ 
surge using GnRH agonist in an antagonist down-regulated IVF cycle. Hum Reprod. 2003;18(10):2079-2081.

30. Castillo JC, Dolz M, Bienvenido E, Abad L, Casan EM,
Bonilla-Musoles F. Cycles triggered with GnRH agonist: exploring low-dose HCG for luteal support. Reprod Biomed Online. 2010;20(2):175-181. 